\title{
LAS COMPLEJIDADES DE LA SEGURIDAD Y LA SOBERANÍA ALIMENTARIA EN VENEZUELA
}

\author{
Francisco Domínguez \\ Middlesex University
}

\section{LAS COMPLEJIDADES DE LA SEGURIDAD Y LA SOBERANÍA ALIMENTARIA EN VENEZUELA}

Resumen: La seguridad y la soberanía alimentarias que normalmente están íntimamente ligadas lo están aun más en Venezuela debido a las características económicas estructurales de esta economía petrolera y, desde 1999, debido a las transformaciones en las que se embarcó esta nación a partir de la inauguración de la Revolución Bolivariana iniciada por Hugo Chávez Frías. La justicia social, especialmente la erradicación de la pobreza, así como también todas las secuelas asociadas con esta lacra tan común en América Latina, tales como la informalidad laboral, la exclusión socio-política de amplias capas de la población pobre e indigente, y sobre todo la mortalidad infantil, la desnutrición y la malnutrición, han sido blancos y objetivos privilegiados del proceso Bolivariano. De allí que la seguridad alimentaria haya sido proclamada como un derecho constitucional, específicamente el Art.305 de la Constitución de la República Bolivariana de Venezuela consagra el Derecho a la Alimentación como política fundamental de Estado. Los logros en seguridad alimentaria son de destacar, obteniendo Venezuela Bolivariana ya dos reconocimientos oficiales de la FAO. Sin embargo, los logros en soberanía alimentaria, pese a existir son más modestos, siendo el obstáculo principal la estructura económica de alta especialización petrolera por más de un siglo de la economía de esta nación sudamericana. Un segundo obstáculo más contemporáneo pero no por ello menos serio en la consecución no sólo de la soberanía sino que especialmente de la seguridad alimentaria, es la guerra económica, que como en el Chile de Salvador Allende, busca erosionar la base de apoyo político del bolivarianismo por medio del acaparamiento, el contrabando de alimentos, artículos de primera necesidad y combustibles en gran escala, especulación en divisa, y la utilización del fracking para inducir los precios del crudo a niveles de desplome como arma política contra gobiernos y regímenes que Washington detesta. Y, como en Chile, tales planes de desestabilización pueden ser letales.

Palabras clave: Seguridad alimentaria, soberanía alimentaria, Venezuela, petróleo, agricultura, bolivarianismo, desnutrición infantil, guerra económica, exclusión social, pobreza, alimentos.

\section{THE SECURITY COMPLEXITIES AND FOOD SOVEREIGNTY IN VENEZUELA}

Abstract: Security and food sovereignty are usually closely linked, even more so in Venezuela, but due to the structural economic characteristics of its oil economy, and, since 1999, due to the transformations in which this nation embarked from the onset of the Bolivarian Revolution initiated by Hugo Chavez Frias. Social justice, especially poverty eradication, and all the consequences associated with this scourge so common in Latin America, such as labor informality, socio-political exclusion of large sections of the poor and destitute, and, above all, child mortality, malnutrition and undernourishment, have been priority objectives of the Bolivarian process. Because of that, food security has been proclaimed as a constitutional right, specifically Art.305 of the Constitution of the Bolivarian Republic of Venezuela enshrines the right to food as a fundamental state policy. The achievements in food security are noteworthy, leading Bolivarian Venezuela to merit two FAO official recognitions. However, the achievements in food sovereignty are more modest, the main obstacle being the century-old highly specialized structure around oil of this South American nation's economy. A second obstacle, more contemporary but no less serious in achieving not only sovereignty but especially food security is the economic war that, as in the Chile of Salvador Allende, seeks to erode the base of political support for bolivarianism through hoarding, smuggling food staples and fuel, large-scale currency speculation, and the use of fracking to induce oil prices to collapse levels as a political weapon against governments and regimes that Washington detest. And, as in Chile, such destabilisation plans can be lethal.

Key words: Food security, food sovereignty, Venezuela, oil, agriculture, bolivarianism, child malnutrition, economic war, social exclusión, poverty, foods. 


\section{INTRODUCCIÓN}

En Venezuela Bolivariana, el derecho a la alimentación no es una abstracción a la que se aspira sino un área de actividad estatal y gubernativa vigorosa que ha significado no sólo la creación de un Ministerio para la Alimentación sino que además, ha llevado al establecimiento de la Misión Alimentación, institución nacional dependiente del Ministerio que está incluida en el Plan de la Patria 2013-2019 plan económico matriz del gobierno del Presidente Nicolás Maduro - dentro del Sector Agricultura, Tierras y Alimentación. A todo ello se debe agregar varias cadenas y redes de producción alimentaria así como de distribución de propiedad social que realizan una labor permanente procurando garantizar que la ciudadanía, especialmente su mayoría pobre, tenga acceso a todos los artículos de la canasta básica a precios tanto regulados como subsidiados y con ello, dar cumplimiento concreto al principio del derecho a la alimentación consagrado en la Constitución Bolivariana de 1999.

Si se tratase sólo de aumentar la producción agrícola y pecuaria del país y de adoptar políticas de distribución justa para que la mayoría más pobre vea realizado su derecho a la alimentación consagrado en la constitución, una revolución como la Bolivariana, tendría relativamente pocas dificultades para conseguirlo, sin embargo, la especialización económica exacerbada de extracción y exportación de petróleo de esta nación, ha distorsionado su economía de tal manera que la realización del derecho a la alimentación se enfrenta a una cantidad insoluble de obstáculos estructurales objetivos. Pero aun, la política de la soberanía alimentaria depende de otra política proclamada por la constitución bolivariana, la soberanía alimentaria, un objetivo aún más difícil de llevar a cabo.

En este artículo intentamos explicar cómo, pese a las enormes dificultades estructurales objetivas, Venezuela Bolivariana ha obtenido logros extraordinarios en relación a la seguridad alimentaria de su población, lo que ha ido de la mano con políticas de redistribución de la renta petrolera que han reducido la pobreza a sus niveles históricos más bajos en décadas. Al mismo tiempo procuramos analizar las complejidades asociadas a la soberanía alimentaria, cuya solución incompleta dificulta y precariza la realización de la seguridad alimentaria de la mayoría pobre de Venezuela.

Por último, daremos algunos datos clave sobre la guerra económica desencadenada por una coalición de fuerzas externas y domésticas desde la prematura partida de Hugo Chávez en marzo de 2013 y que, como en el Chile de Salvador Allende, apuntan a debilitar y desmoralizar las bases sociales, políticas y electorales de apoyo al proceso de transformación revolucionaria en curso en Venezuela, y que, como en Chile, buscan crear las condiciones para el derrocamiento del gobierno bolivariano, antesala de la erradicación del chavismo tanto en Venezuela como en el resto de América Latina.

\section{LA MALDICIÓN AGRÍCOLA DEL PETRÓLEO: el} desarrollo del subdesarrollo de la agricultura

Venezuela se transformó de una economía esencialmente ganadera y productora de cacao en 1920 a una economía casi completamente dominada por la extracción y exportación del petróleo. De la misma manera que Venezuela se convirtió en una economía altamente especializada -de casi dedicación exclusiva- en la exportación de petróleo, también se convirtió en consecuencia en una economía altamente importadora de todo lo que se consumía tanto en artículos de primera necesidad y de lujo así como de materias primas intermedias y procesadas además de maquinaria y vehículos.

En la Tabla 1 se puede ver que, en gran medida como consecuencia del surgimiento de la industria del petróleo, Venezuela prácticamente vació sus campos en el que es probablemente el proceso de urbanización más acelerado y más intenso de América Latina puesto que entre 1941 y 2001 (60 años) su población rural descendió de $68,7 \%$ a apenas un $12,3 \%$, es decir un descenso de $60 \%$, mientras que su población urbana creció en el mismo período de $31,3 \%$ a $87,7 \%$, es decir casi un $60 \%$. Este proceso no se he detenido y hoy (2016) Venezuela tiene una población urbana, altamente concentrada en unas ocho grandes ciudades, de aproximadamente $96 \%$, lo que significa que apenas un $4 \%$ de la población es rural.

Tabla 1 - Población rural y urbana en diversos censos 1941-2001 (porcentajes)

\begin{tabular}{l|c|c}
\hline Año & $\begin{array}{l}\text { Población } \\
\text { urbana }\end{array}$ & Población rural \\
\hline 1941 & 31,3 & 68,7 \\
\hline 1950 & 47,4 & 52,6 \\
\hline 1961 & 62,1 & 37,9 \\
\hline 1971 & 72,8 & 27,2 \\
\hline 1981 & 80,3 & 19,7 \\
\hline 1990 & 84,1 & 15,9 \\
\hline 2001 & 87,7 & 12,3 \\
\hline
\end{tabular}

Fuente: INSTITUTO NACIONAL DE ESTATISTICA. Censo de Población y Viviendas de 2001. Madrid, 2001.

Las consecuencias de la altísima especialización económica petrolera para el desarrollo integral de la nación han sido devastadoras. Citamos in extenso el informe de J. L. Hernández ([2006]) en el que se señala: 


\begin{abstract}
La expansión petrolera generó un cambio fundamental en la distribución territorial del poder y la riqueza. La dinámica económica, basada en la importación, el comercio, los servicios, la construcción y el sector público, se centró en las ciudades, mientras que las zonas rurales permanecieron estancadas 0 en franco deterioro. El poder articulado a los sectores agroexportadores fue también afectado por la depresión de los años treinta. El sostenido crecimiento económico posterior se interrumpió a finales de la década de los 70 , cuando se inició un período de inestabilidad, recesión y aumento de la pobreza, la inseguridad alimentaria y la exclusión social de vastos sectores de la población. Desde la primera crisis cambiaria, en 1983, los períodos de ajuste y de generación de desequilibrios se han repetido cíclicamente, con independencia de la orientación del gobierno de turno. (HERNÁNDEZ, [2006], p. 7).
\end{abstract}

La sobre especialización petrolera produjo un padrón de comportamiento cíclico de la economía venezolana que esencialmente desde la crisis cambiaria de 1983 consistió en un período de expansión (causado por el aumento del precio y la demanda del petróleo) seguida de un período de ajuste económico (que incluían ajuste cambiario y fiscal), seguido a su vez por una recesión (caída del Producto Interno Bruto (PIB), alta inflación y caída en las importaciones), que a su vez llevaban a un desajuste económico (caracterizado por sobreevaluación cambiaria y expansión del gasto público, seguida por otro periodo de expansión económica.

Por todo lo señalado, la producción agrícola de Venezuela al momento del ascenso de Hugo Chávez al gobierno en 1999 y desde la década de los 80 se ubicaba en alrededor de un $5 \%$ del PIB nacional con leve tendencia a la disminución, probablemente una de las más bajas en América Latina, y cuya distribución era la siguiente (HERNÁNDEZ, [2006]): Las granjas avícolas y porcinas integradas representan alrededor del $27 \%$ de PIB agrícola con un número reducido de productores. La labranza mecanizada moderna, productora de cereales y oleaginosas de ciclo corto, más del $18 \%$ del PIB agrícola y un gran número de productores y asalariados. Las haciendas ganaderas de leche y cría, $15 \%$ del PIB y alta generación de empleo permanente. La cadena de producción ganadera de cría, levante y ceba, $14 \%$ del producto agrícola, pero con bajo número de productores y trabajadores. Otros sistemas importantes son la pequeña producción frutícola y hortícola, la pequeña y mediana producción cafetalera y cacaotera y los complejos agroindustriales azucareros y de palma aceitera.
Muchos dirigentes políticos e intelectuales pensaban que el futuro de Venezuela estaba en el campo o en el uso de la renta petrolera para financiar la industrialización y modernización del país. Ya en 1936 el prestigioso escritor Arturo Uslar Pietri (1936, p. 2) publicó en el periódico Ahora su famoso articulo Sembrar el petróleo en el que señala:

Urge aprovechar la riqueza transitoria de la actual economía destructiva para crear las bases sanas y amplias y coordinadas de una futura economía progresiva que será nuestra verdadera acta de independencia. Es menester sacar la mayor renta de las minas para invertirla totalmente en ayudas, facilidades y estímulos a la agricultura, la cría y las industrias nacionales. Que en lugar de ser el petróleo una maldición que haya de convertirnos en un pueblo parásito e inútil, sea la afortunada coyuntura que permita con su súbita riqueza acelerar y fortificar la evolución productora del pueblo venezolano en condiciones excepcionales. [...] La única política económica sabia y salvadora que debemos practicar, es la de transformar la renta minera en crédito agrícola, estimular la agricultura científica y moderna, importar sementales y pastos, repoblar los bosques, construir todas las represas y canalizaciones necesarias para regularizar la irrigación y el defectuoso régimen de las aguas, mecanizar e industrializar el campo, crear cooperativas para ciertos cultivos y pequeños propietarios para otros.

La transformación económica estructural de Venezuela, sin embargo, fue al revés, de una economía agraria a una petrolera, de una sociedad rural a una hiper-urbanizada ocurrió en apenas un par de décadas y cuya profundidad no puede exagerarse. En el bien documentado trabajo sobre la modernización agrícola de Venezuela, Robertina García (1991, p. 1), nos da las cifras de tal transformación:

El desplazamiento del predominio agroexportador por el petroexportador queda demostrado por la correspondencia que tienen ambos componentes en loa años posteriores al descubrimiento del petróleo [...] en el lapso 1915-20, los productos agrícolas aportaban el $96 \%$ del total exportado, con elevada participación del café (51.8\%), descendiendo sostenidamente en los años que prosiguen, hasta ubicarse en apenas $3.5 \%$ en el periodo $1945-50$. A mismo tiempo, la exportación petrolera crece aceleradamente desde $0.6 \%$ al comienzo del quinquenio revisado hasta $88 \%$ en $1930-35$, desacelerando luego su ritmo hasta situarse en $96 \%$ en la mitad de la década de los años cincuenta. 
En su monografía García (1991) traza el proceso de abandono de la agricultura por parte del estado, hacia la cual los gobiernos aplican paliativos (devaluación para proteger a la agricultura, subsidios a la actividad agrícola exportadora y tasas de cambio diferenciales) que no fueron adecuados para abordar el meollo estructural del problema: la industria del petróleo puede desarrollarse con un tipo de cambio alto, que otorga al bolívar un mayor poder adquisitivo externo, mientras que la agricultura no tiene posibilidades de sobrevivir si no se beneficia de robustas medidas proteccionistas.

En concreto esto significó la excesiva dependencia de las exportaciones de petróleo, que se benefician de un bolívar alto, aumentando el poder adquisitivo de la nación, en tanto que para la agroexportación un bolívar alto significa una menor cantidad de bolívares a cambio de sus divisas que no alcanzan a cubrir sus costos.

Inexorablemente esta dinámica creó desempleo y bajísimos estándares de vida en el campo, en las ciudades, donde se concentra la explotación petrolera, tuvo su corolario demográfico en una hipertrofia urbana y el subdesarrollo de la ya subdesarrollada agricultura nacional, con sus secuelas de ineficiencia, bajísima productividad y poco atractivas tasas de ganancia, pero especialmente incapacidad estructural para satisfacer la demanda nacional de alimentos. Así un aumento de la demanda de alimento de una creciente población urbana, enfrenta un sector agrícola cuya oferta es totalmente insuficiente, creando con ello déficits alimentarios que deben ser suplidos con una creciente importación. Gracias a su alta rentabilidad, la industria del petróleo convierte a Venezuela en un neto importador de alimentos ya en 1940.

La renta petrolera, la maldición económica de Venezuela y la fuente de muchas de sus ventajas pero también muchos de sus problemas, no se usó para la modernización del país sino hasta 1945 cuando el presidente Betancourt fomentó la economía productiva apoyando al capital privado a través de generosos préstamos y apoyos financieros a empresas molineras de trigo, lácteas, azucareras y muchas otras. Sin embargo, pese al apoyo estatal a la agricultura, especialmente en las décadas de 1950 y 1970, incluyendo un substancial aumento de la mecanización, la utilización de fertilizantes el aumento del crédito agrícola e incluso la creación de varios bancos para este efecto (Banco Agrícola y Pecuario y Banco de Desarrollo Agropecuario, por ejemplo), que produjo mejoras temporales pero tenues en la producción alimentaria, que resultaron no ser sostenibles en el mediano plazo. En este período, la substitución de importaciones beneficia principalmente a la industria manufacturera y sólo marginalmente a la agricultura dado que beneficia a la industria elaboradora de alimentos pero no así a la producción agrícola, excepto en rubros tales como cereales y oleaginosas.

Como consecuencia del agotamiento de la industrialización por medio de la substitución de importaciones que además del débil crecimiento de los precios agrícolas conjuntamente con la robustez del crecimiento de los insumos para la agricultura (maquinaria, fertilizantes, etc.), ya para 1970 hay aumentos substanciales en las importaciones de rubros tales como carne de bovino, maíz, leguminosas y frutas frescas y elaboradas. Así por ejemplo, la importación de rubros esenciales para el consumo de la mayoría de la población aumentó entre los años 1960 y 1979. Para las caraotas de 12 a 21 millones de kg, la de frijoles de 2 a casi 5 millones de kg, la de maíz de 15 a 374 millones de kg, y, por último, la de azúcar de 1.6 a casi 189 millones de $\mathrm{kg}$. Todo ello en un contexto de descenso sostenido y dramático de la población rural y de aumento inexorable de la población urbana que para 1979 era ya $83 \%$ mientras que la rural era apenas $17 \%$. Esto significó que las importaciones agrícolas subieron de 2.101 millones de bolívares en 1972 a 5.376 millones de bolívares en 1979 (GARCÍA, 1991). Entre 1970 y 1979 la superficie total cultivada para cereales, leguminosas, oleaginosas, tubérculos, frutas, hortalizas, y otros declinó de un total de 1828.0 a 1742.8 miles de hectáreas (Ministerio de Agricultura y Cria apud GARCIA, 1991).

En la década de los 80 Venezuela seria afectada, tardíamente, por la oleada neoliberal que acuciaba al resto del continente desde los 1970 y durante la cual el PIB por habitante decrece a una tasa promedio de $-24.9 \%$ para el período 1980-89 (COMISIÓN ECONÓMICA PARA AMÉRICA LATINA Y EL CARIBE, 1996). La política cambiaria fue modificada y la política de control de precios de artículos de consumo alimentario fue substancialmente relajada restringiéndose así a 11 el número de productos protegidos (arroz, maíz, sorgo, girasol, soya, copra, aceite semirefinado de palma, caña de azúcar, café, cacao, leche y semillas), 13 productos menos que en 1974. Todo ello conlleva aumentos significativos de los precios de artículos como arroz, maíz, sorgo, aunque desde 1980 la Corporación de Mercado Agrícola (CMA) introdujo subsidios para artículos importados como el arroz, maíz, sorgo, maní, ajonjolí, soya y leche, subsidios que, debido a la caída del la renta petrolera, se adopta una política de su eliminación progresiva (GARCIA, 1991). Peor aún, el sistema de cambios múltiples, del cual la agricultura se beneficiaba del cambio preferencial, fue substituido por un sistema de cambio único y flotante. La aplicación del Plan de Ajuste Estructural (PAE) en febrero de 1989 llevaría a un desplome del producto del sector $(-6.5 \%$ para al sector agrícola vegetal, $-11.2 \%$ el animal, $-8.2 \%$ para el pesquero). Todo ella produce un cuadro acercándose al catastrófico puesto que 
[A] partir de 1984, cambia el rumbo de todos los índices, con incrementos para 1988 de 9 veces en lo que a los productos agrícolas respecta, 7 veces los salarios, 15 veces la maquinaria y algo más del doble para los fertilizantes. (GARCIA, 1991, p. 25, 36). ${ }^{1}$

\section{LA INCREÍBLE PESADEZ DEL LEgADO PETROLERO RENTISTA}

En Notas de Análisis, Juan L. Hernández ([2006]) describe el estado socio-económico de la mayoría de la población, registrando con ello el enorme lastre que dejó la IV República y su secuela de pobreza, exclusión social y deficiencia alimentaria de millones de venezolanos:

El largo período de dificultades económicas generó un amplio proceso de empobrecimiento y de exclusión social de vastossectores de la población. En los períodos en que se agudiza el deterioro social, tras cada crisis económica, se instrumentaron medidas para mitigar sus efectos y combatir la pobreza. Sin embargo, en todos los casos éstas han resultado insuficientes para contener los efectos de la dinámica económica.

La capacidad adquisitiva de los salarios se deteriora gravemente a partir de la década de los ochenta. En 1999 los salarios reales representan sólo el $40 \%$ de los existentes en 1980 y su capacidad adquisitiva en alimentos registra incluso un nivel más bajo. El proceso continuó al menos hasta 2003, cuando las remuneraciones de los trabajadores, en términos reales, se ubican en menos del $70 \%$ de las recibidas en 1997. (HERNÁNDEZ, [2006], p. 11).

En el grafico (Figura 1) se puede apreciar

Figura 1 - Salario real y capacidad adquisitiva en alimentos 1968-1999 (base $100=$ 1968)

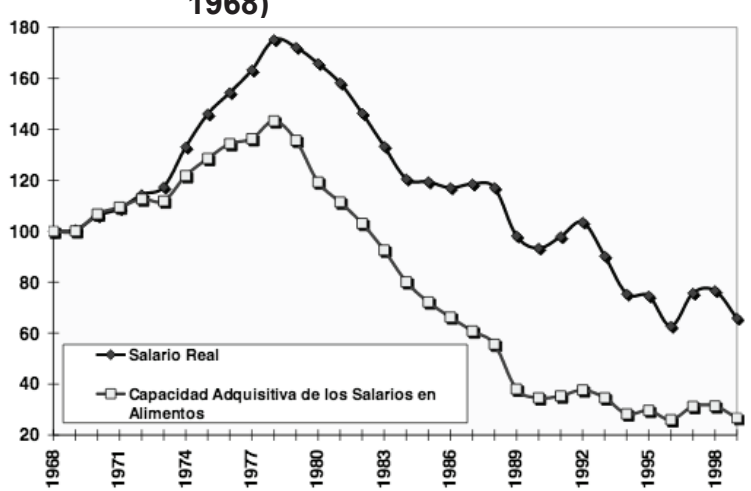

Fonte: Elaboración del autor, con base em los dados de: ORGANIZACIÓN DE LAS NACIONES UNIDAS PARA LA AGRICULTURA Y LA ALIMENTACIÓN; COPORACIÓN ANDINA DE FOMENTO. Venezuela: nota de análisis sectorial - agricultura y desarollo rural. Roma, [20--?]. que luego de un peak de 160 (tomando 1968 como año base $=100$ ) a mediados de los 1970, tanto el salario real como su capacidad adquisitiva en alimentos declinó sostenidamente, con el primero a 50 mientras que el segundo declinaba catastróficamente a un poco más de 20 puntos. En otras palabras, la exclusión social tenia una relación funcional a la incapacidad de amplios sectores de la población de obtener seguridad alimentaria.

La situación de postración social, económica, política y moral en que la IV República dejó a la nación en 1998 fue tan profunda que casi no tiene precedentes en el resto de la región que para entonces pasaba por un período de intensas convulsiones como consecuencia de 30 años de políticas neoliberales. Esta realidad es registrada por todos los análisis y monografías publicadas sobre el surgimiento del chavismo a partir de El Caracazo en 1989, la insurrección cívico-militar fallida de 1992, y la elección Hugo Chávez a la presidencia de Venezuela en 1998. Tal punto de vista lo comparten incluso intelectuales pro-neoliberales fuertemente asociados con la derecha nacional e internacional como Ricardo Hausmann y Francisco Rodríguez (2014 traducción libre del autor), quienes en Venezuela Before Chavez, que tiene el elocuente subtítulo de Anatomía de un Desplome Económico, señalan:

En 1979 esa tendencia [de crecimiento económico sostenido que el país había experimentado desde el comienzo del siglo $X X]$ iba a sufrir un giro de $180^{\circ}$. El PIB per cápita del sector no petrolero declinó a una tasa anual de 0.9 por ciento durante los próximos veintitrés años, en un total acumulado de 18.6 por ciento. Lo que es aún más notable es que esto ocurrió pese a una significativa incorporación de nuevos trabajadores en la fuerza laboral, que debería ceteris paribus haber incrementado su ingreso per cápita. Por lo tanto, el PIB por trabajador cayó a una tasa anual de 1.9 por ciento en el sector no petrolero; su declive acumulado fue de 35.6 por ciento.

El puntojijismo (nombre peyorativo de la IV República) se caracterizaba por su excesiva corrupción en un sistema de falsa democracia dirigida para fines de clase muy estrechos por una muy bien estructurada y coherente parasitocracia organizada en dos partidos políticos - COPEI y ADECO - que se habían turnado en el poder desde 1958 y cuya finalidad casi exclusiva era embolsarse una parte substancial de la renta petrolera y garantizar un barril de petróleo bajo como condición para recibir el apoyo político, estratégico, financiero, y cultural de Estados Unidos. Las consecuencias de tan nefasto y excluyente sistema produjeron el desplome de la nación en todo orden de áreas de la vida social y política. El mecanismo clave de 
los privilegios y el poder de la parasitocracia era su control sobre Petróleos de Venezuela (PDVSA), empresa estatal del petróleo, la empresa que administra la renta petrolera proveniente de las exportaciones de los hidrocarburos. La reproducción de los privilegios y el poder de la parasitocracia tenia su corolario inexorablede pobreza y exclusión social de sectores cada vez más amplios de la población. Un experto venezolano en la industria del petróleo, Carlos Mendoza Pottellá (1995, p. 1), en monografía galardonada, que procura "[...] demostrar el carácter antagónico al interés nacional de los emprendimientos del poder del petróleo.", señala que la industria del petróleo

[...] en 1991, por ejemplo, ocupando directamente a 42.911 personas, el $0,6 \%$ de la población económicamente activa, haya generado 673 mil millones de bolívares de producto, equivalentes al 22,2 por ciento del PTB nacional, de los cuales se recabó del 80 por ciento de los ingresos fiscales ordinarios y originaron el $81,6 \%$ de las exportaciones. $\mathrm{Y}$, como es sabido, éstas han sido relaciones prácticamente constantes durante los últimos 30 años, por lo menos y así lo podemos observar por ejemplo, en el cuadro "Petróleo y Desarrollo" que anualmente publica el Ministerio de Energía y Minas en las primeras páginas del PODE: para 1969, con 24.521 empleados directos, el $0,8 \%$ de la población económicamente activa de entonces, la industria petrolera generaba el $22,3 \%$ del PTB, el $63,3 \%$ de los ingresos fiscales y el $91,7 \%$ de las exportaciones. (POTTELLÁ, 1995, p. 14 , grifo do autor).

Es decir, una diminuta proporción de venezolanos obtenía empleo e ingreso de la renta petrolera, que sólo beneficiaba a un grupo minúsculo de la parasitocracia que se enriquecía con el patronaje que ejercía sobre PDVSA, lo que a su vez, le permitía el control sobre el gobierno y sobre todo el aparato de estado. Un historiador británico ha descrito PDVSA como una

[...] compañía que ha sido administrada por años como una empresa corporativa, un estado dentro del estado, un vasto conglomerado que distribuía favores y sobornos. (GOTT, 2011, p. 170). ${ }^{2}$

La pesadez del legado puntofijista consistía no sólo de un inmenso déficit social que excluía de todo, no sólo de la economía, sino también de la política, la cultura, la educación y toda otra área de significancia, a millones de venezolanos, sino de que además, del legado de una estructura económica que aunque ha paradójicamente permitido al estado bolivariano enfrentar las lacras sociales asociadas a la IV República, de manera creciente se ha ido convirtiendo en el gran obstáculo que impide progresar más allá de los efectos beneficiosos dela política redistributiva. Aunque estos beneficiosos son substanciales y reales (reducción de la pobreza e indigencia, expansión de la educación y la salud gratuita, provisión de vivienda a millones, expansión y mejora de los sistemas de transporte público, acceso a materiales escolares y tecnológicos gratuitos, elevación de los niveles culturales de la población, alimentos subsidiados y tantas otros más), debido a que dependen de la renta petrolera, no pueden ser garantizados en el largo plazo.

Tal estructura económica tan altamente especializada para la exportación de hidrocarburos permite que se trastornen y se compliquen con mucha facilidad los sistemas de distribución comercial de artículos de primera necesidad tales como el alimento. Adicionalmente, una caída violenta y sostenida del precio y demanda del petróleo debilita la moneda nacional lo que la hace altamente vulnerable a la especulación cambiaria así como también a la de artículos de primera necesidad y el acaparamiento de éstos últimos permite sobreprecios y especulación en el mercado negro, además de contrabando, todo lo cual redunda en presiones inflacionarias galopantes. En realidad, la hiper-especialización petrolera de Venezuela ha sido históricamente una maldición que llevo a Juan Pablo Pérez Alfonzo (1976), Ministro de Minas e Hidrocarburos de Venezuela en 1959-1964 y además, fundador de la Organization of Petroleum Exporting Countries (OPEC), en un famoso libro suyo se refirió al petróleo como el excremento del diablo.

El cuadro socio-económico de Venezuela en 1999 era el siguiente: la economía decreció en un $7.20 \%$ y entre la pobreza, que afectaba al $17,65 \%$ de los hogares en 1980, aumentó a un asombroso $48,33 \%$, mientras que las cifras para la pobreza extrema fueron de $9,06 \%$ y $27,66 \%$ respectivamente. Si el nivel de desempleo era $10.30 \%$ en 1983 , y había subido ligeramente a $11,30 \%$ en 1998 , lo que en verdad existía era desempleo disfrazado de empleo informal que en 1983 haba sido 41,30\% y que para 1998 había alcanzado el 48,20\% (MAYA, 2005). El desempleo entre los jóvenes de 15 a 24 años era 18,9\% en 1999 (VENEZUELA, 2015). El gasto público se contrajo de $37 \%$ del PIB en 1982 a $16 \%$ del PIB en 1998 lo que, conjuntamente con la devaluación del bolívar en 100\% entre 1988 y 1993 afectó negativamente aún más los ya deprimidos estándares de vida de la mayoría de los venezolanos. $Y$ los déficits en la balanza de pagos causados por la volatilidad del mercado del petróleo entre 1986 y 1988 llevó a un creciente endeudamiento de US\$26.6 billones en 1988 a US\$37 billones en 1998 (CANNON, 2009). El índice de prevalencia de subnutrición en 1999 fue de 21 y el porcentaje de niños desnutridos en 1998 fue de 5,30\%, sólo 
$80 \%$ de la población tenía acceso a agua potable en 1980, y la esperanza de vida al nacer en 1998 era de 72 años (VENEZUELA..., 2015).

\section{LA POLÍTICA DE SEGURIDAD ALIMENTARIA Y SUS LOGROS}

Sonestas estructuras y el estado de catástrofe social, económica, política, moral y cultural que el gobierno bolivariano debe enfrentar a fin de abordar tanto la seguridad como la soberanía alimentarias.

El Sistema Socialista de Soberanía Alimentaria en Venezuela incluye un conjunto de instituciones tales como el Ministerio del Poder Popular para la Alimentación (MINPPAL), la Corporación Venezolana Agraria (CVA), la Corporación Venezolana de Alimentos (CORVAL), AGROPATRIA, además de Cooperativas, Empresas de Producción Socialista y Consejos Comunales Agrarios, Casas de Alimentación, Corporación de Abastecimientos y Servicios Agrícolas (CASA), Mercados y Alimentos (MERCAL), Productora y Distribuidora de Alimentos (PDVAL), además de muchos otros organismos que cubren el área de la seguridad y soberanía alimentarias de la nación y del pueblo.

Todas ellas tienen la función de hacer cumplir el mandato de la Constitución Bolivariana de 1999 que estipula en art. $305^{\circ}$ que

[...] el Estado promoverá la agricultura sustentable como base estratégica del desarrollo rural integral a fin de garantizar la seguridad alimentaria de la población. (VENEZUELA, 1999).

En otras palabras el acceso a la alimentación es un derecho constitucional de todos los venezolanos. Hay además los siguientes instrumentos legales al respecto de la agricultura, ganadería, alimentación y servicios relacionados, a saber:

Ley Orgánica de Seguridad y Soberanía Agroalimentaria, la Ley de Salud Agrícola Integral., la Ley de Crédito para el Sector Agrario, la Ley del Banco Agrícola, la Ley de Beneficios y Facilidades de Pago para las Deudas Agrícolas y Rubros Estratégicos para la Seguridad y Soberanía Alimentaria, la Ley para la Defensa de las Personas en el Acceso para los Bienes y Servicios

Además el artículo $5^{\circ}$ de la Ley Orgánica de Seguridad y Soberanía Agroalimentaria explícitamente menciona como objetivos de la seguridad agroalimentaria:

Garantizar el balance alimentario de la población, a través de: la planificación, el desarrollo sistémico y articulado de la producción, así como la promoción de la actividad agropecuaria.
El establecimiento de medidas en el orden financiero, de intercambio y distribución, comercial, transferencia tecnológica, tenencia de la tierra, infraestructura, formación y capacitación, y otras que fueren necesarias, con el fin de alcanzar los niveles de autoabastecimiento requeridos por la población y evaluar el rendimiento de las inversiones, su impacto, la verificación precisa del correcto uso de los recursos públicos invertidos y su efecto económico-social. La protección de los asentamientos y comunidades de pescadores 0 pescadoras artesanales, así como sus caladeros de pesca en aguas continentales y los próximos a la línea de costa definidos en la ley.

Cualquier otra actividad que determine el reglamento del presente Decreto con Rango, Valor y Fuerza de Ley Orgánica. Asegurar la distribución de la producción nacional agroalimentaria con el propósito de atender la satisfacción de las necesidades básicas de la población. (VENEZUELA, 2008).

Además, el Ministerio de Salud ha publicado un plan Política Nacional de Seguridad Alimentaria y Nutricional 2011-2021 que procura garantizar la seguridad alimentaria hasta el año 2021.

La primera medida que Hugo Chávez tomó para resolver la espinuda cuestión de la soberanía y seguridad alimentarias fue redistribuir tanta tierra cultivable como fuese posible a individuos e instituciones que produjeran para reducir el déficit domestico procurando satisfacer la demanda interna y lograr así disminuir la dependencia externa. Así antes de la llegada de Chávez el latifundio tenía 6.762.399 hectáreas, de las cuales hasta 2012 3.654.681 hectáreas habían sido redistribuidas (recuperadas) por Chávez, con 3.107.718 hectáreas más en proceso de recuperación. Es decir, la reforma agraria chavista llevó a la erradicación del latifundio, lacra que ha representado un obstáculo objetivo al desarrollo de la nación, situación que en Venezuela se vio agravada por la mayor ineficiencia relativa de su agricultura comparado con el resto de América Latina (AGENCIA VENEZOLANA DE NOTÍCIAS, [20--?]).

Los resultados del aumento del producto agrícola agregado, debido a las dificultades estructurales mencionadas, fueron insuficientes para eliminar la dependencia de las importaciones, con la complicación adicional de que, debido a las políticas redistributivas y el aumento de la oferta de alimentos subsidiados, le demanda ha continuado sobrepasando la oferta agregada. Por ello, pese a que la producción nacional de alimentos que incluye productos agrícolas, animales y pescados, se incrementó de 13 millones de toneladas en 1998 a 25 millones en 2013, el consumo de alimentos de primera necesidad que, en 1999 había sido de casi 14 millones de toneladas, ya en 2012 superaba las 26 millones de toneladas. La Tabla 2 nos muestra el 
crecimiento de la producción agrícola por rubros al comparar los años 1998 y $2014^{3}$, y es de notar que entre 1990 y 2008 la producción agrícola total creció en un tercio.

Los incrementos en la producción sin una política deliberada y específicamente enfocada a resolver la deficiencia alimentaria de la mayoría de la población, sólo habría probablemente incrementado las exportaciones sin impacto real en la seguridad alimentaria de la población. Los mayores aumentos de la producción de productos agrícolas para la alimentación son precisamente aquéllos de la dieta básica de la mayoría de los venezolanos tales como arroz blanco, maíz amarillo, maíz blanco, papas y azúcar.

Las políticas públicas del gobierno bolivariano: redistribución del ingreso, control de los precios, tres comidas gratuitas a 4 millones de niños en las escuelas, y la distribución de alimentos subvencionados a través de MERCAL y PDVAL, además de la educación de la población en cuestiones de dieta familiar, así como el aumento de la producciónagrícola, han producido resultados bastante positivos.

Para empezar, el consumo individual de los venezolanos en kilo-calorías diarias ha aumentado significativamente: de 2204 en 1990 bajó a 2127 en 1999, para aumentar a 3290 en 2012 (en el 2015, como consecuencia de las secuelas de la guerra económica se ubica en 3092). Esto ha ido acompañado de un substancial aumento del consumo de leche que de 44 litros per cápita en 1999 pasó a 120 en 2010, cantidad que se espera continúe incrementándose en el futuro.

Así por ejemplo, el índice de la prevalencia de la subnutrición que en 1990-1992 era de 11\%, y que para el período 1998-2000 había aumentado a $21 \%$, para el período $2008-2010$ había declinado drásticamente a apenas $2 \%$. El índice más positivamente afectado con las políticas de soberanía y seguridad alimentarias fue la desnutrición infantil que pasó de 7,66 en 1990 a 2,9 en 2011, es decir una reducción de más del $60 \%$. De la misma manera, la proporción de niños y niñas menores de 5 años con peso inferior al normal que en 1990 había sido $7.70 \%$, en 2012 había bajado a $2.73 \%$.

Las cifras macroeconómicas confirman las

Tabla 2 - Producción agrícola - años selectivos tendencias positivas de estos índices: el 95.4\% de los venezolanos come tres y más veces al día; más de 4 millones de niños y niñas comen dos comidas y una merienda en las escuelas bolivarianas; 900 mil personas reciben una comida en 6000 casas de alimentación; y el gobierno da además un ticket de alimentación que en 2014 era de 0,75 Unidades Tributarias por día laboral. La FAO ha registrado el hecho de que en Venezuela 4.717.372 personas han dejado de padecer hambre. Por todo esto, Venezuela está entre los 5 países de América Latina con mayor avance en la erradicación del hambre, y además es el cuarto país de la región con un adecuado suministro alimentario para la satisfacción de las necesidades nutricionales de su pueblo (AGENCIA VENEZOLANA DE NOTÍCIAS, [20--?]).

El 16 de mayo de 2015, el representante de FAO, Marcelo Rosende, elogió los esfuerzos y avances de Venezuela en materia de seguridad y soberanía alimentarias. Ya el 27 de noviembre de 2014, la FAO había hecho declaraciones de felicitaciones a Venezuela por la erradicación del hambre. El representante de FAO que participara en la cumbre de la CELAC, Adoniram Sánchez, dijo que "[...] en lo político y en lo estadístico el hambre es un tema superado en Venezuela." (FAO..., 2014). Además el director regional de FAO, Raúl Benítez elogió a Hugo Chávez diciendo que la

[...] erradicación del hambre era la mejor forma que tenemos para honrar a este hombre que fue tan visionario en este tema. Se adelantó 10 años al resto de los líderes mundiales y lo hizo efectivo. (FAO..., 2014).

En la 39 Conferencia de la FAO en Roma, Italia, en junio de 2015, la FAO otorgó un reconocimiento a Venezuela. Jorge Arreaza, vicepresidente de Venezuela, precisó que desde 2003 se han distribuido más de 25 millones de toneladas de alimentos de la canasta básica y se ha atendido a más de 22 millones de venezolanos y que se han invertido US\$142 mil millones en seguridad alimentaria; agregó que para la Revolución Bolivariana la lucha contra el hambre y la lucha para "[...] garantizar la alimentación de todos los ciudadanos es una de la directrices y de las líneas fundamentales." (VENEZUELA..., 2015).

\begin{tabular}{c|l|c|c|c|c|c|c|c}
\hline $\begin{array}{c}\text { Año } \\
\text { (en toneladas) }\end{array}$ & Cereales & $\begin{array}{c}\text { Granos y } \\
\text { leguminosas }\end{array}$ & $\begin{array}{c}\text { Textiles y } \\
\text { oleaginosas }\end{array}$ & $\begin{array}{c}\text { Raíces y } \\
\text { tubérculos }\end{array}$ & Frutales & Hortalizas & $\begin{array}{c}\text { Cultivos tropicales } \\
\text { tradicionales }\end{array}$ & Total \\
\hline 1990 & 1.780 .246 & 59.666 & 500.674 & 625.749 & 2.602 .738 & 400.060 & 6.725 .394 & 12.694 .617 \\
\hline 1998 & 2.133 .160 & 33.601 & 569.416 & 1.053 .896 & 2883.663 & 896.196 & 8.208 .162 & 15.778 .093 \\
\hline 2008 & 4.014 .492 & 27.916 & 614.889 & 1.024 .160 & 2.540 .428 & 885.929 & 8.782 .249 & $17,890.063$ \\
\hline 2014 & 3.364 .063 & 14.509 & 572.566 & 1.038 .927 & 4.018 .341 & 1.340 .367 & 6.055 .812 & 16.404 .585 \\
\hline
\end{tabular}

Fonte: Compilación del autor: VOLUMEN de producción por grupos del subsector vegetal Venescopio: Venezuela en cifras, Caracas, [2009]. Disponível em:<http://www.venescopio.org.ve/estadisticasbasicasdevenezuela/volumen-de-produccion-por-grupos-delsubsector-vegetal>. Acceso en: 4 ene. 2016; CONFEDERACIÓN DE ASOCIACIONES DE PRODUCTORES AGRÍCOLAS. Indicadores de la producción por grupo de cultivo. Caracas, [20--?]. Disponível em:<http://www.fedeagro.org/produccion/ default.asp>. Acceso en: 4 ene. 2016. 


\section{LA GUERRA ECONÓMICA, EL PEOR OBSTÁCULO OBJETIVO A LA SOBERANÍA Y SEGURIDAD ALIMENTARIAS}

No hay duda que desde aproximadamente 2012 Venezuela ha estado sometida una guerra económica que evoca los aciagos años de Salvador Allende en Chile en los 1970. El gobierno ha denunciado con evidencia convincente de que sectores empresariales en complicidad con la derecha de ese país por medio del acaparamiento para deliberadamente causar escasez de artículos de primera necesidad, especialmente alimentos e incluyendo medicamentos, que el estado venezolano había importado con dólares preferenciales - a 6.30 Bolívares - y había puesto en circulación a precios subsidiados; el contrabando en gran escala a Colombia de estos mismos artículos, beneficiándose de enormes diferenciales de precios entre las dos economías, particularmente gasolina; especulación en el mercado negro de estos rubros también en gran escala, impulsando la inflación, ya de por si alta; y, también en escala gigantesca, especulación cambiaria con divisas, que, debido a la tasa venezolana de cambio fijada artificialmente baja para reducir el impacto de la inflación en los niveles de vida de la población, otorga niveles de ganancia estratosféricos. A todo esto debe agregarse la caída inducida, principalmente por el fracking, del precio del petróleo en más de 100 dólares el barril.

La evidencia disponible sugiere que todas estas actividades forman parte de un plan de desestabilización que procura debilitar el apoyo popular a la Revolución bolivariana orientado, como en el caso de Allende en Chile, al derrocamiento del gobierno bolivariano presidido por Nicolás Maduro. Entre muchas otras evidencias están por un lado, la incautación por las fuerzas del orden de miles de toneladas de alimentos, de artículos de primera necesidad, e incluso de productos médicos, y millones de galones de gasolina, pero por otro, están los literalmente cientos de reportajes especiales, documentales y artículos de los principales medios de comunicación corporativos del mundo que, en un tono más de regocijo que de curiosidad periodística, lo confirman. El gobierno bolivariano ha, desde 1999, denunciado intentos de derrocamiento violento por parte de una coalición de fuerzas nacionales e internacionales, dirigidas desde el Depto. de Estado en Washington y señalan una cantidad substancial de tales intentos, incluyendo la injerencia estadounidense en los asuntos internos de Venezuela por medio, entre muchos otros, del financiamiento de partidos de la oposición, medios de comunicación, asociaciones cívicas de oposición que se presentan como ONGs, financiamiento que en algo así como una década un total que supera los US\$100 millones ${ }^{4}$. El impacto negativo de la guerra económica se ha hecho sentir en la caída de la producción de alimentos en los rubros claves afectando a los artículos de consumo masivo lo que es un hecho desde 2012-2013.

En conclusión, la experiencia bolivariana en Venezuela entre 1998-2015 demuestra que es posible adoptar políticas públicas que garanticen la seguridad alimentaria de toda la población, especialmente de aquéllos millones de ciudadanos excluidos por el neoliberalismo precedente, y particularmente de millones de niños. También, esta experiencia demuestra que se puede lograr avances substanciales en obtener la soberanía alimentaria. Sin embargo, la altísima especialización de la economía venezolana en la exportación de petróleo junto a la volatilidad contemporánea de esta commodity, ponen en peligro la regularidad de los recursos en el mediano y sobre todo en el largo plazo y con ello amenaza los logros obtenidos. De la misma manera, las secuelas de escasez en la distribución de alimentos causados por la guerra económica reducen y amenazan los logros en la seguridad y soberanía alimentarias. La conclusión inevitable es que, para economías como las latinoamericanas, el control estatal sobre materias primas claves es una condición necesaria para financiar la seguridad y soberanía alimentarias por medio de la reforma agraria, incentivos a la producción e importación de alimentos a precios y tasas preferenciales a los rubros de alimentos. Pero una condición suficiente, es una robusta presencia estatal en el área de la distribución y comercialización de los alimentos. Por último, el adverso resultado de la elección a la Asamblea Nacional el 6 de diciembre de 2015 en Venezuela en que el descontento con las consecuencias de la guerra económica ha producido una mayoría parlamentaria de la derecha cuya filosofía y política amenazan, no sólo la seguridad y soberanía alimentarias, sino todos los logros obtenidos en 16 años de bolivarianismo.

\section{REFERENCIAS}

AGENCIA VENEZOLANADE NOTÍCIAS. Venezuela socialista en cifras. Caracas, [20--?]. Disponible em:<http://venezuelasocialista.avn.info.ve/>. Acceso en: 4 ene. 2016.

ALFONZO, J.P.P. Hundiéndonos en el excremento del diablo. Caracas: Editorial Lisbona, 1976.

CANNON, B. Hugo Chavez and the Bolivarian

Revolution: populism and democracy in a globalised age. Manchester: Manchester University Press, 2009.

COMISIÓN ECONÓMICA PARA AMÉRICA LATINA $Y$ EL CARIBE. Transferencia productiva en equidad: la tarea prioritaria de América Latina y el Caribe en los años noventa. Santiago de Chile, 1996. 
CONFEDERACIÓN DE ASOCIACIONES DE PRODUCTORES AGRÍCOLAS. Indicadores de la producción por grupo de cultivo. Caracas, [20--?]. Disponível em:<http://www.fedeagro.org/ produccion/default.asp>. Acceso en: 4 ene. 2016.

FAO: El hambre en Venezuela es tema superado. Telesur, nov. 2014. Disponível em:<http://www. telesurtv.net/news/FAO--El-hambre-en-Venezuelaes-tema-superado-20141127-0062.html>. Acceso em: 4 ene. 2016.

GARCIA, R. El Proceso de modernización agrícola en Venezuela. 1991. Trabalho de Conclusão de Curso (Monografia) - Insituto Interamericano de Cooperación para la Agricultura, Caracas, 1991.

GOTT, R. Hugo Chavez and the Bolivarian Revolution. Brooklyn: Verso Books, 2011.

HAUSMANN, R.; RODRÍGUEZ, F. (Eds.). Venezuela before Chavez: anatomy of an economic collapse. University Park, PA: Pennsylvania State University Press, 2014. Primera página del capítulo 1 Why Did Venezuela Growth Collapse?

HERNÁNDEZ, J. L. Venezuela: nota de análisis sectorial - agricultura y desarrollo rural. Roma: FAO/ CAF, [2006]. Disponible en:<ftp://ftp.fao.org/docrep/ fao/012/ak170s/ak170s00.pdf>. Acceso en: 3 ene. 2016.

INSTITUTO NACIONAL DE ESTATISTICA. Censo de Población y Viviendas de 2001. Madrid, 2001.

MAYA, M. L. Del viernes negro al referendo revocatório. Venezuela: Editorial Alfa, 2005.

ORGANIZACIÓN DE LAS NACIONES UNIDAS PARA LA AGRICULTURA Y LA ALIMENTACIÓN; COPORACIÓN ANDINADE FOMENTO. Venezuela: nota de análisis sectorial - agricultura y desarollo rural. Roma, [20--?].

PIETRI, A. U. Sembrar el petróleo. Ahora, Venezuela, ano 1, n. 183, jul. 1936. Disponible en:<http://prof. usb.ve/emendoza/orosas/212/Textos/Sembrar_el_ petroleo.pdf>. Acceso en: 31 dic. 2015.

POTTELLÁ, C. M. El Poder Petrolero y la Economía Venezolana. Caracas: LITOPAR, 1995. Disponível em:<http://petroleovenezolano.blogspot. co.uk/2009/12/universidad-central-de-venezuela. html>. Acceso en: 2 ene. 2016.

UNITED STATES. Covert Action in Chile 19631973: staff report on the Select Committee to Study Governmental Operations with Respect to Intelligence Activities. Washington, DC: Government
Printing Office, 1975.

VENEZUELA recibe reconocimiento de la FAO por lucha contra el hambre. Globovision, [S. I.], jun. 2015. Disponível em:<http://www.dailymotion.com/ video/x2t607c>. Acceso en: 4 ene. 2016.

VENEZUELA. Constitución de la República Bolivariana de Venezuela. Caracas, 1999. Disponível em:<http://www.mp.gob.ve/LEYES/ constitucion/constitucion1.html>. Acceso en: 4 ene. 2016.

VENEZUELA. Ministerio del Poder Popular de Planificación. Venezuela en cifras: nuestra transición al socialismo. Caracas, feb. 2015. Disponíble en:<http://www.mppp.gob.ve/libro/mayo pdf/VzlaEnCifras\%2005-2015.pdf>. Acceso en: 4 ene. 2016.

VENEZUELA. República Bolivariana de Venezuela. Ley Orgánica de Seguridad y Soberanía Agroalimentaria. Gaceta Oficial, Caracas, 2008.

VOLUMEN de producción por grupos del subsector vegetal Venescopio: Venezuela en cifras, Caracas, [2009]. Disponível em:<http://www.venescopio. org.ve/estadisticasbasicasdevenezuela/volumende-produccion-por-grupos-del-subsector-vegetal>. Acceso en: 4 ene. 2016.

\section{NOTAS}

EI PAE de febrero de 1989 llevó a la explosión social conocida como El caracazo que quiebró el espinazo de la IV República, creando las condiciones del surgimiento del fenómeno Hugo Chavez, la Revolución Bolivariana y la V República.

2 Otros autores que en el mundo anglo-sajón coinciden con esta descripción de la dinámica diabólica del estado petrolero-parásito que domino la vida, la economía, la sociedad y la política por más de 40 años llevándola finalmente al colapso son, sin ningún orden específico, Barry Cannon, Hugo Chavez and the Bolivarian Revolution. Populism and Democracy in a Globalised Age, Manchester University Press, 2009; Bart Jones, The Hugo Chavez Story, The Bodley Head, 2008; Gregory Wilpert, Changing Venezuela by Taking Power, Verso 2006; Steve Ellner and Miguel Tinker Salas (editors), Venezuela: Hugo Chavez and the decline of an 'exceptional democracy', Rowman \& Littlefield Publishers, 2006; Michael Derham, Politics in Venezuela: Explaining Hugo Chavez, Peter Lang AG, 2010; Nikolas Kozloff, Hugo Chavez: Oil, Politics, and the Challenge to the U.S., Palgrave Macmillan, 2007; Miguel Tinker Salas, Venezuela, What Everyone Needs to Know, Oxford University Press, 2015; Buxton, Julia (2001) The Failure of Political Reform in Venezuela, Aldershot, England: Ashgate; Coronil, Fernando (1997) The Magical State, Chicago: University of Chicago Press; Ellner, Steve and Daniel Hellinger (eds.) (2003) Venezuelan Politics in the Chávez Era: Class, Polarization, and Conflict, London: Lynne Rienner 
Publishers; Gott, Richard (2000) In the Shadow of the Liberator, republicado como: Hugo Chavez and the Bolivarian Revolution for the expanded 2005 edition. London: Verso Books; Karl, Terry Lynn (1997) The Paradox of Plenty, Berkeley: University of California Press; Lebowitz, Michael (2006) Build it Now: Socialism for the 21st Century New York: Monthly Review Press; McCoy, Jennifer and David Myers (eds.) (2004) The Unraveling of Representative Democracy in Venezuela, Baltimore: Johns Hopkins University Press; y Raby, D.L. (2006) Democracy and Revolution: Latin America and Socialism Today, London: Pluto Press.

3 Cifras compiladas de: Venesocpio (VOLUMEN..., [2009]) y Confederación de Asociaciones de Productores Agrícolas ([20--?]).

4 No debemos olvidar que el Informe Oficial de EE.UU. sobre la Acción Estadounidense Encubierta en Chile 1963-1973, se señala que entre las acciones desestabilizadoras contra Allende incluyeron 'hacer chillar la economía' - en la famosa frase del Presidente Richard Nixon -, propaganda mediática negativa, apoyo a los medios de comunicación de y partidos políticos de la oposición, subvencionar 'asociaciones cívicas', y apoyo al sector empresarial, esfuerzos directos para influencias a los militares. Véase Covert Action in Chile 1963-1973, Staff Report on the Select Committee to Study Governmental Operations with Respect to Intelligence Activities (UNITED STATES, 1975).

\section{Francisco Domínguez}

Economista Político

Doctor em Economia Política da La United Kingdon

Head of the Latin American Studies Research Group and

Senior Lecturer in Spanish in Middlesex University

E-mail: f.dominguez@mdx.ac.uk

\section{Middlesex University}

Hendon campus - The Burroughs, London, NW4 4BT 